\title{
Minimally Invasive Surgery for Intra-Articular Calcaneus Fractures: A 9-year, Single-Center, Retrospective Study of A Standardized Technique Using A 2- Point Distractor
}

\author{
Christian Rodemund \\ Unfallkrankenhaus Linz \\ Ronny Krenn \\ Unfallkrankenhaus Linz \\ Carl Kihm \\ Norton Audubon Hospital \\ Iris Leister \\ Paracelsus Medizinische Privatuniversitat \\ Reinhold Ortmaier \\ Ordensklinikum Linz GmbH Barmherzige Schwestern \\ Werner Litzlbauer \\ Unfallkrankenhaus Linz \\ Angelika Schwarz \\ Unfallkrankenhaus Graz https://orcid.org/0000-0003-4369-9133 \\ Georg Mattiassich ( $\sim$ georg.mattiassich@gmx.at) \\ Teaching Hospital of the Medical University Graz https://orcid.org/0000-0002-5743-6783
}

\section{Research article}

Keywords: Calcaneal Fracture, Minimally Invasive, Intra-articular Fractur

Posted Date: July 2nd, 2020

DOI: https://doi.org/10.21203/rs.3.rs-37201/v1

License: (c) (i) This work is licensed under a Creative Commons Attribution 4.0 International License. Read Full License

Version of Record: A version of this preprint was published on November 14th, 2020. See the published version at https://doi.org/10.1186/s12891-02003762-9. 


\section{Abstract}

\section{Background:}

A fracture of the calcaneus can be a painful and disabling injury. Treatment modalities may be conservative or operative, as in open reduction and internal fixation (ORIF) via different approaches, including a number of minimally invasive methods. The aim of this study was to evaluate the treatment options and post-treatment complication rates for intra-articular calcaneal fractures at the Traumacenter Linz over a 9-year period.

\section{Methods:}

All patients with calcaneal fractures treated at the Traumacenter Linz between 2007 and 2016 were included in this study. The patients' protocols were retrospectively reviewed, and the data, including demographic parameters, cause of injury, and the time between the accident and the first operative treatment were analyzed. Secondary operative interventions due to soft-tissue complications, hardware removal, and the long-term arthrodesis rate were evaluated.

Results:

A minimally invasive 2-point-distractor method was used in $85.8 \%(n=182)$ of all operatively managed calcaneal fractures ( $n=236)$ in our department. The majority of the operations (88.7\%) were performed within two days after the accident. The secondary operation rate resulting from wound complications was $2.7 \%$ in the 2-point distractor group and $16.7 \%$ in the ORIF group. A secondary arthrodesis was performed in $4.7 \%$ ( $n=9$ ) of the subtalar joints in the entire study population.

\section{Conclusions:}

Our data supported the assumption that severe wound complications would be less likely to occur after minimally invasive treatment compared to ORIF treatment. Additionally, our arthrodesis rate was comparable to that in the literature.

Trial Registration: Not applicable, level of evidence IV.

\section{Background}

A calcaneal fracture can be a painful and disabling injury. Fractures of the heel bone account for about $2 \%$ of all fractures, and are often intra-articular, multi-fragmentary, and comminuted. Standardized treatment protocols are lacking, and the optimal treatment of intra-articular calcaneal fractures is still controversial. Multiple factors such as fracture pattern, comorbidities, timing, and status of the soft tissue must be considered. (1-3)

Treatment modalities vary between conservative, open reduction and internal fixation (ORIF), numerous minimally invasive approaches, and even primary subtalar joint arthrodesis. (4-6)

Prolonged and eventful healing or mal-reduction of the fracture can lead to a poor result and persistent disability. $(3,7)$ The goal of operative management is to achieve an anatomical reduction of the joint surfaces and a reconstruction of the length, width, height, and axis of the calcaneus. However, an anatomic reconstruction cannot always be achieved in severely comminuted fractures. $(6,8)$ Optimal treatment minimizes operative soft tissue dissection, which reduces the risk of wound dehiscence and does not limit adjunctive procedures. $(3,9)$ If a secondary arthrodesis of the subtalar joint is required later, the procedure is generally easier to perform after a previous minimally invasive procedure. This will lead to a better clinical result since the calcaneal axis has already been corrected, and the bone stock has been remodeled. $(5,10)$

Accounting for the advantages of operative treatment in general, and minimally invasive treatment in particular, we implemented a treatment protocol in our department and standardized the operative technique from positioning to X-ray views, repositioning and osteosynthesis, and postoperative care.

The aim of this study was to evaluate this treatment protocol and the subsequent complication rate in the management of intra-articular calcaneal fractures over a 9-year period. Herein, we also describe our technique (see additional file 1).

\section{Methods}

\section{Study design and participants}

A retrospective data-analysis was performed on the data of 298 patients at the Traumacenter Linz, Austria. Between 1/1/2007 and 1/1/2016, patients with uni- or bilateral open or closed calcaneal fractures, treated operatively or conservatively with a follow up of at least 12 months were included in the study. Patients were analyzed using the medical documentation system and the medical controlling data of the Austrian Social Insurance for Occupational Risks (AUVA).

Demographic data, cause of injury, time from injury to operation, treatment modality, need for revision surgery and complications were analyzed.

The study protocol was approved by the ethics committee of the AUVA, and was performed in accordance with the protocol (Votum 10/2016). 
Herein, we describe our operative techniques and protocol for the management of open or closed calcaneal fractures (see additional file 1). Written informed consent was obtained from patients to illustrate the method.

\section{Complications}

Complications were defined as postoperative wound healing problems that required revision surgery, unplanned hardware removal due to irritation of the soft tissue, or the need for a subtalar arthrodesis. An elective hardware removal was not considered to be a complication. These procedures were performed with the intention to avoid interference with a potential arthrodesis in the future or after based on an explicit request from the patient.

\section{Statistical Analysis}

Descriptive statistics, including means and standard deviations (SDs) for continuous variables (age, time from accident to operative intervention) and frequency counts for categorical variables (sex, treatment modality, trauma mechanism, operative technique, infectious complications, number of implant removals, cases necessitating secondary subtalar arthrodesis) were calculated.

All statistical analyses were performed using SPSS statistical software (version 23, SPSS Inc., Chicago, Illinois, USA), and p<.05 indicated a statistically significant result.

\section{Results \\ Demographics}

From 2007 to 2016, 298 patients with calcaneal fractures treated at the Traumacenter Linz were identified. Of these, 236 were men (79.2\%) and 62 were women $(20.8 \%)$. In total, 212 patients $(71.1 \%)$ were treated operatively, and 86 patients $(28.9 \%)$ were treated conservatively. The ages ranged from 15 to 82 years. The mean age of the operatively treated patients was 45.7 (range, 15-79) years, whereas that for the conservatively treated patients was 44.2 (range, 7-94) years. Details see Table 1.

\section{Trauma mechanism}

The most common injuries were ground-level falls (29\%), occurring mostly in elderly female patients. Falls from a height of more than 2 meters occurred in $30 \%$ of cases, mostly in young male patients. External trauma, such as from motor vehicle accidents, occurred in $33 \%$ of these patients.

\section{Operative technique}

The operative technique is given to supplement this article. Within the 9-year period, 212 patients were operatively treated at the Traumacenter Linz, and the majority were treated using the 2-point distraction method (182 patients, $85.8 \%$ ); a patient case is displayed in Fig. 1 . Only 7 patients were treated with ORIF (3.3\%). K-wire fixations, or combinations of plates and K-wires with or without utilizing the 2-point distractor were performed on 23 patients (10.8\%). The annual distribution of the operative techniques is presented in Table 2.

\section{Time from accident to operative intervention}

The operative interventions were performed within the first 2 days after injury in $88.7 \%$ of the patients. Most of the patients with later surgery times (314 days) were transferred from other hospitals or had multiple injuries with delayed treatment of their calcaneal fractures.

\section{Complications}

Due to the low number of patients that underwent ORIF, their data were pooled with that of the group treated with K-wires or a combination of K-wires and plates (30 patients) to compare with the minimally invasive operations group (182 patients).

Wound complications that required revision occurred in 4.7\% (10 patients). Five patients were in the minimally invasive group, representing a $2.7 \%$ infection risk in this group, and 5 patients were in the other group, representing $16.7 \%$ in that group. Specifics see Table 3.

Unplanned hardware removals due to irritation of the soft tissue or adjacent joints were performed in $4.2 \%$ of the study population. The secondary subtalar arthrodesis rate was $4.7 \%$ in the whole collective at the time of the data analysis (June 2017). No primary arthrodesis was performed. Elective hardware removals were performed in $34.9 \%$ of all patients due to subjective irritation of the screws, at patient request, or under the consideration that a secondary subtalar arthrodesis could interfere with the arthrodesis. The sustentaculum screw was left in situ in almost $90 \%$ of the patients. An overview of the performed implant removals and secondary subtalar fusions is presented in Table 4.

The rate of unplanned revision surgeries between 2007 and 2010 was higher (7.3\%) than that in the 2011-2015 period (1.7\%). The rate of elective hardware removal between 2007 and 2010 was $41.7 \%$ compared to 29.3\% between 2011 and 2015. These numbers can be attributed to the increasing

Page $3 / 10$ 
experience with this technique and the modification of screw positioning.

\section{Discussion}

We have modified and standardized the technique of minimally invasive operative treatment of intra-articular calcaneal fractures using the 2-point distractor through changes in positioning of the patient, intraoperative radiological viewing, screw placement adjustments, and aftercare. We believe that this technique is advantageous for ORIF in 3 respects. The main advantage of this technique is the reduced rate of wound complications (2.7\%) compared to ORIF (16.7\%) in our study population. Another important benefit is the possibility of performing the procedure immediately without waiting for the consolidation of the soft tissue. This primarily affects the patient since an immediate operation reduces the pressure on the soft tissue and consequently reduces the level of pain, and it is of socioeconomic interest as the time in the hospital for detumescence is reduced. Last but not least, the overall arthrodesis rate of $4.7 \%$ at the Traumacenter Linz is comparable to the literature, although we treat all types of fracture morphologies via minimally invasive means, regardless of the amount of comminution. Arthrodesis is technically easier after minimally invasive procedures.

Many studies have been published concerning the optimal method of treating intra-articular calcaneal fractures. $(1-3,6,9,11-15)$ Most of them lacked a representative number of patients, and therefore, a general consensus is still undetermined. $(1,2,14)$

At the Traumacenter Linz, the demographic analysis revealed relatively young patients (mean 43.3 years) and a male predominance of 3.8:1, which reflects the high socioeconomic influence on this fracture occurrence rate. Causes of injury were high-energy trauma in the majority of patients. Compared to the international literature, falls from heights are the most likely causes of injuries according to Mitchell et al. (16) and Alexandridis et al. (17) Bohl et al. (18) reported a lower incidence of falls, but a higher rate of traffic accidents (49\%).

Open reduction and internal fixation (ORIF) has been the preferred therapy for intra-articular fractures. $(1,2)$ The generally accepted approach for visualization of the fracture site is the extended L-shaped lateral approach, which is considered to be the gold standard. (1) Independent of the approach in ORIF, a consolidation of the soft tissues is recommended. $(2,5,19)$ However, it can take $2-4$ weeks before the so-called "wrinkle-sign," and decreased swelling occurs. $(2,5)$ Al-Mudhaffar et al. reported an increased incidence of wound healing problems when the operative procedures were performed within the first week after the injury in an open setting. (19) Rammelt et al. concluded that an operative intervention after 2 weeks also increased the complication rate, which could be explained by increasing fracture consolidation prior to surgery and a resulting need for higher force with reduction. (5) In our described technique, the operation is intended to be performed within the first 3 days after injury. Even with edematous tissue, our method did not lead to an increase in wound healing problems. The earlier the operation was performed, the easier the mobilization of the fragments became.

To overcome problems with wound complications, wound infections, and skin necrosis specifically, many minimally invasive methods to reduce and fix calcaneal fractures have been proposed. (15) At the Traumacenter Linz, this rate was $2.7 \%$ using the 2-point distraction method. We believe this is not only a consequence of the operative technique, but also a result of early operative intervention with hematoma evacuation, reduction, and stabilization. This leads to a decrease in the internal pressure.

In a meta-analysis, Fan et al. compared the clinical results after minimally invasive techniques to those after ORIF. Fan et al. reported a lower soft tissue complication rate and reduced duration of the operative procedure. Functional results were almost equivalent for the two groups. (13)

The minimally invasive technique is a standardized procedure at the Traumacenter Linz, and about $86 \%$ of all operatively treated fractures have been utilized in the 2-point distractor technique.

Comparisons between the different methods of minimally invasive techniques and ORIF methods are difficult due to a lack of standardized measures, different techniques, and a low number of patients. $(12,15,20,21)$

Wallin et al. published a systematic review on the clinical results after minimally invasive techniques used to treat calcaneal fractures. The functional results after Sanders type II-IV compared with ORIF were promising, although most of the studies had low evidence levels. Soft tissue complications and duration of the procedure were lower in the minimally invasive group. They did not discuss whether minimally invasive techniques or ORIF led to better anatomic reductions and functional results. (15)

In a randomized, controlled study, Kumar et al. found a lower rate of wound healing problems and better functional outcomes in the minimally invasive group, compared with those after ORIF. The better functional outcomes were explained by a lower wound complication rate and a better anatomical reconstruction. (22) In terms of anatomic reconstruction, ORIF is still considered the gold standard for intra-articular fractures. $(1,2,9,13)$

In 2007, Schepers et al. presented their results on minimally invasive methods with a follow-up of 3 years. Functional results after minimally invasive techniques were lower compared to those reported after ORIF. The infection and wound complication rates were similar to those of ORIF. Subtalar joint motion could be restored to nearly $70 \%$ compared to the uninjured side. The secondary arthrodesis rate was higher than in Buckley's study from 2002.

It seems that the most crucial factor for gaining a satisfactory result, according to Veltman, is the absence of complications. (1) Also, other authors concluded that patients show the best results when the operative procedure and aftercare were complication-free. $(2,3)$ Patients with comorbidities such as vascular diseases, diabetes, and nicotine abuse are more prone to these perioperative complications. (2) Also, patients over 60 years old are more likely to be affected by postoperative complications and subtalar arthritis, although this may be linked to the higher rate of comorbidities. (2)

Page $4 / 10$ 
The published rate of arthrodesis is between 0 and $15 \%$ after minimally invasive surgery: $0-12 \%$ after ORIF, and 3.8-17\% after conservative means. (23-32) The subtalar arthrodesis rate of $0 \%$ described by Park et al. was based on a relatively low number of patients and only one year follow-up. (29) In extremely complex intra-articular fractures, a primary subtalar arthrodesis is postulated to achieve satisfactory results. (2)

The secondary subtalar osteoarthritis rate requiring arthrodesis after minimally invasive techniques was $4.7 \%$. Between 2007 and 2015 , this arthrodesis rate was almost constant. A primary subtalar arthrodesis was not performed in our group of patients. The secondary arthrodesis rate of $4.7 \%$ after operative intervention in our study is comparable to that of the current literature. In a study published by Buckley et al., 37 of 218 (16.9\%) patients required an arthrodesis, whereas 7 of 206 operatively-treated patients (3.4\%) required a secondary operative intervention. (33) More prospective randomized studies with longer follow-up periods are required to compare the results of minimally invasive techniques with ORIF and conservative treatment.

After conservatively treated calcaneal fractures, a 6-fold higher likelihood of arthrodesis has been published compared to primarily operated patients. Furthermore, patients with Sanders Type IV and patients with a Boehler angle of 0 degrees had a notably increased risk of secondary subtalar arthrodesis. (34) Operative reconstruction after calcaneal fractures does provide a better basis for a secondary subtalar arthrodesis and does lead to better long-term results. (9)

Functional results after minimally invasive treatment are equivalent to those of ORIF. $(35,36)$ Based on these functional results with lower complication rates, two recent sets of authors have argued that minimally invasive techniques are a better option for the treatment of intra-articular fractures. (13, 15)

\section{Limitations}

This study has certain limitations and weaknesses that must be considered.

First and foremost, we have no clinical evaluations with comparative scores for our patients. This study was entirely focused on the rate of wound complications, the need for secondary arthrodesis, and relevant characteristics such as demographic data and socioeconomic data.

Future comparative studies are needed to verify the safety of operative procedures for calcaneus fractures. Whether this new technique will result in satisfactory long-term outcomes or can prevent post-traumatic osteoarthritis was not fully determined.

\section{Conclusions}

We believe that minimally invasive procedures for the treatment of intra-articular calcaneus fractures can provide several benefits. These techniques lead to satisfactory results when the whole process from clinical and radiological examination, to indication, positioning, X-ray views, reduction techniques, stabilization methods, and aftercare treatments are performed in a standardized fashion. The main advantage of our proposed technique is the low rate of wound complications compared to ORIF.

\section{Abbreviations}

Open reduction and internal fixation (ORIF)

Kirschner-wires (K-Wires)

Minimal invasive technique (MIT)

Austrian Social Insurance for Occupational Risks (AUVA)

\section{Declarations}

\section{Ethics approval and consent to participate}

The study protocol was approved by the ethics committee of the Austrian Social Insurance for Occupational Risks (AUVA), and was performed in accordance with the protocol (Votum 10/2016).

\section{Consent for publication}

Not applicable.

\section{Availability of data and materials}

The datasets used and/or analyzed during the current study are available from the corresponding author at a reasonable request.

\section{Competing interests}

The authors declare that they have no competing interests. 


\section{Funding}

The project was supported by a research grant from the Austrian Social Insurance for Occupational Risks (AUVA). The grant was used for the design of the study and collection, patient administration, analysis, and interpretation of data and in writing as well as editing the manuscript.

\section{Authors contributions}

CR: Conception and design, collection and assembly of data, analysis and interpretation of the data, figure design, writing of the manuscript.

RK: Conception and design, collection and assembly of data, drafting of the article, critical revision of the article for important intellectual content.

CK: Conception and design, drafting of the article, critical revision of the article for important intellectual content, approval of the manuscript.

IL: Analysis and interpretation of the data, collection and assembly of data, drafting of the article, preparation and review of the manuscript.

RO: Concept and design, analysis of data, preparation and review of the manuscript.

WL: Collection and assembly of data, interpretation of results, preparation and review of manuscript.

AS: Analysis and interpretation of the data, figure design, preparation and review of manuscript.

GM: Idea of the study, figure design, interpretation of results, critical revision of the article for important intellectual content, final approval of the manuscript.

All authors agreed on the interpretation of the results. All authors were involved in the drafting of the manuscript and approved the final version to be submitted for publication.

\section{Acknowledgements}

We would like to thank Editage (www.editage.com) for English language editing.

\section{Authors informations}

Homepage: www.calcaneal-fracture.com

Christian Rodemund, MD ${ }^{1}$; Georg Mattiassich, MD ${ }^{6 *}$.

\section{References}

1. Veltman ES, Doornberg JN, Stufkens SA, Luitse JS, van den Bekerom MP. Long-term outcomes of 1,730 calcaneal fractures: systematic review of the literature. J Foot Ankle Surg. 2013;52(4):486-90.

2. $10.1016 /$ j.fas. 2015.10 .003

Sharr PJ, Mangupli MM, Winson IG, Buckley RE. Current management options for displaced intra-articular calcaneal fractures: Non-operative, ORIF, minimally invasive reduction and fixation or primary ORIF and subtalar arthrodesis. A contemporary review. Foot Ankle Surg [Internet].

2016;22(1):1-8. Available from: http://dx.doi.org/10.1016/j.fas.2015.10.003.

3. Gougoulias N, Khanna A, McBride DJ, Maffulli N. Management of calcaneal fractures: Systematic review of randomized trials. Br Med Bull. 2009;92(1):153-67.

4. Rammelt S, Gavlik JM, Zwipp H. Historical and current treatment of calcaneal fractures. J Bone Joint Surg Am [Internet]. 2001;83-A(9):1438; author reply 1439-40. Available from: http://jbjs.org/content/83/9/1438.abstract.

5. Rammelt S, Zwipp H. Calcaneus fractures: Facts, controversies and recent developments. Injury. 2004;35(5):443-61.

6. Meena S, Gangary SK, Sharma P. Review, Article. Operative versus non- operative treatment for displaced intra- articular calcaneal fracture : a metaanalysis of randomised controlled trials. J Orthop Surg. 2016;24(3):411-6.

7. 10.1053/j.jfas.2013.04.002

Veltman ES, Doornberg JN, Stufkens SAS, Luitse JSK, van den Bekerom MPJ. Long-term outcomes of 1,730 calcaneal fractures: Systematic review of the literature. J Foot Ankle Surg [Internet]. 2013;52(4):486-90. Available from: http://dx.doi.org/10.1053/j.jfas.2013.04.002.

8. Ibrahim T, Rowsell M, Rennie W, Brown AR, Taylor GJS, Gregg PJ. Displaced intra-articular calcaneal fractures: 15-Year follow-up of a randomised controlled trial of conservative versus operative treatment. Injury. 2007;38(7):848-55.

9. Guerado E, Bertrand ML, Cano JR. Management of calcaneal fractures: What have we learnt over the years? Injury. 2012;43(10):1640-50.

10. Radnay CS, Clare MP, Sanders RW. Subtalar Fusion After Displaced Intra-Articular Calcaneal Fractures: Does Initial Operative Treatment Matter? J Bone Jt Surgery-American Vol. 2010 Mar;92(Suppl 1):32-43.

11. Epstein N, Chandran S, Chou L. Current concepts review: intra-articular fractures of the calcaneus. Foot Ankle Int. 2012;33(1):79-86. 
12. Schepers T, Patka P. Treatment of displaced intra-articular calcaneal fractures by ligamentotaxis: Current concepts' review. Arch Orthop Trauma Surg. 2009;129(12):1677-83.

13. Fan B, Zhou X, Wei Z, Ren Y, Lin W, Hao Y, et al. Cannulated screw fixation and plate fixation for displaced intra-articular calcaneus fracture: A metaanalysis of randomized controlled trials. Int J Surg. 2016;34:64-72.

14. Jiang N, Lin QR, Diao XC, Wu L, Yu B. Surgical versus nonsurgical treatment of displaced intra-articular calcaneal fracture: A meta-analysis of current evidence base. Int Orthop. 2012;36(8):1615-22.

15. Wallin KJ, Cozzetto D, Russell L, Hallare DA, Lee DK. Evidence-based rationale for percutaneous fixation technique of displaced intra-articular calcaneal fractures: A systematic review of clinical outcomes. J Foot Ankle Surg. 2014;53(6):740-3.

16. Mitchell MJ, McKinley JC, Robinson CM. The epidemiology of calcaneal fractures. Foot (Edinb). 2009 Dec;19(4):197-200.

17. Alexandridis G, Gunning A, van Olden G, Verleisdonk E, Segers M, Leenen L. A Trauma System wide Evaluation of the Demographic, Injury and Fracture Characteristics of Patients with Calcaneal Fractures: A Comparison of Trauma Level I and II Centers. Clin Res Foot Ankle. 2017;5(2).

18. Bohl DD, Nathaniel T, Samuel AM, Diaz-collado PJ, Stephen J, Basques BA. A Study of 14516 Patients in the American College of Surgeons National Trauma Data Bank. Foot Ankle Spec. 2016;10(5):402-10.

19. Al-Mudhaffar M, Prasad CV, Mofidi A. Wound complications following operative fixation of calcaneal fractures. Injury. 2000;31(6):461-4.

20. Pelliccioni AAA, Bittar CK, Zabeu JLA. Surgical treatment of intraarticular calcaneous fractures of sanders' types II and III. Systematic review. Acta Ortop Bras [Internet]. 2012;20(1):39-42. Available from: http://www.pubmedcentral.nih.gov/articlerender.fcgi?

artid $=3718410$ \&tool $=$ pmcentrez\&rendertype $=$ abstract $\% 5 \mathrm{Cn} \% 22 \% 5 \mathrm{C} \% 5 \mathrm{Cschbs} 02 \% 5 \mathrm{Cdaten} \% 5 \mathrm{CReferenceManager} \% 5 \mathrm{CPublikationen} \% 5 \mathrm{Cp} / \mathrm{Pellicioni}$ 2012_Surgical treatment of intraarticular.pdf\%22.

21. Bhattacharya R. Sanders classification of fractures of the os calcis: AN ANALYSIS OF INTER- AND INTRA-OBSERVER VARIABILITY. J Bone Jt Surg Br Vol. 2005;87-B(2):205-8.

22. Sampath Kumar V, Marimuthu K, Subramani S, Sharma V, Bera J, Kotwal P. Prospective randomized trial comparing open reduction and internal fixation with minimally invasive reduction and percutaneous fixation in managing displaced intra-articular calcaneal fractures. Int Orthop. 2014;38(12):2505-12.

23. Buckley R, Tough S, Mccormack R, Pate G, Leighton R, Petrie D, et al. Operative Compared With Nonoperative Treatment of Displaced Intra-Articular Calcaneal Fractures. J Bone Jt Surgery-American Vol. 2002;84(10):1733-44.

24. Schepers T, Schipper IB, Vogels LMM, Ginai AZ, Mulder PGH, Heetveld MJ, et al. Percutaneous treatment of displaced intra-articular calcaneal fractures. J Orthop Sci. 2007;12(1):22-7.

25. Stulik J, Stehlik J, Rysavy M, Wozniak A. Minimally-invasive treatment of intra-articular fractures of the calcaneum. J Bone Jt Surg - Br Vol. 2006;88$B(12): 1634-41$.

26. Tomesen T, Biert J, Frolke JP. Treatment of displaced intra-articular calcaneal fractures with closed reduction and percutaneous screw fixation. J Bone Joint Surg Am. 2011;93(10):920-8.

27. Vroome SW, De LFM, Van Der. Cohort Study on the Percutaneous Treatment of Displaced Intra-articular Fractures of the Calcaneus. Foot Ankle Int. 2014;35(2):156-62.

28. Tantavisut S, Phisitkul P, Westerlind BO, Gao Y, Karam MD, Marsh JL. Percutaneous Reduction and Screw Fixation of Displaced Intra-articular Fractures of the Calcaneus. Foot Ankle Int. 2017;38(4):367-74.

29. Park J, Che JH. The sinus tarsi approach in displaced intra-articular calcaneal fractures. Arch Orthop Trauma Surg. 2017;137(8):1055-65.

30. Ebraheim N, Elgafy H, Sabry FF, Freih M, Abou-Chakra IS. Sinus tarsi approach with trans-articular fixation for displaced intra-articular fractures of the calcaneus. Foot ankle Int / Am Orthop Foot Ankle Soc [and] Swiss Foot Ankle Soc. 2000;21(2):105-13.

31. Griffin D, Parsons N, Shaw E, Kulikov Y, Hutchinson C, Thorogood M, et al. operative versus non-operative treatment for closed, displaced, intraarticular fractures of the calcaneus: randomised controlled trial. BMJ. 2014;349(July):g4483.

32. Agren P, Wretenberg P. AS-N. Operative Versus Nonoperative Treatment of Displaced Intra-Articular Calcaneal Fractures: A Prospective, Randomized, Controlled Multicenter Trial. J Bone Jt Surg. 2013;95(15):1351-7.

33. BUCKLEY R, TOUGH S, MCCORMACK R, PATE G, LEIGHTON R, PETRIE D, et al. Operative Compared With Nonoperative Treatment of Displaced IntraArticular Calcaneal Fractures. J Bone Jt Surgery-American Vol. 2002;84(10):1733-44.

34. Csizy M, Buckley R, Tough S, Leighton R, Smith J, McCormack R, et al. Displaced intra-articular calcaneal fractures: variables predicting late subtalar fusion. J Orthop Trauma. 2003;17(2):106-12.

35. Hoeve S, Van PM. The Journal of Foot \& Ankle Surgery Outcome of Minimally Invasive Open and Percutaneous Techniques for Repair of Calcaneal Fractures: A Systematic Review. J Foot Ankle Surg. 2016;55(6):1256-63.

36. Giannini S, Cadossi M, Mosca M, Tedesco G, Sambri A, Terrando S, et al. Minimally-invasive treatment of calcaneal fractures: A review of the literature and our experience. Injury. 2016;47(November):138-46.

\section{Tables}




\begin{tabular}{|llllllll|}
\hline \multirow{7}{*}{ Procedure } & Sex & N & Min & Max & Median & Mean & SD \\
\hline OP & Men & 174 & 16 & 79 & 46 & 45.3 & 13,5 \\
& Women & 38 & 15 & 73 & 49.5 & 48.0 & 12,5 \\
\hline \multirow{2}{*}{ Conservative } & Men & 62 & 15 & 82 & 42.5 & 44.0 & 18,9 \\
& Total & 212 & 15 & 79 & 46 & 45.7 & 13,3 \\
& Women & 24 & 7 & 94 & 46.5 & 44.7 & 23,7 \\
\hline & Total & 86 & 7 & 94 & 44.5 & 44.2 & 20,3 \\
\hline
\end{tabular}

SD: standard deviation; OP: operative; Min: minimum; Max: maximum

Table 1: Demographics of the patients with a calcaneus fracture treated at the Traumacenter Linz from 2007 to 2016 . In total $n=298$ patients were observed, $71 \%(n=212$ / 298) were treated operatively, $29 \%$ underwent conservative care. An odds ratio of 3:1 (male: female) could be interpreted in both patient groups with a balanced age distribution.

\begin{tabular}{|llllllll|}
\hline \multicolumn{3}{|c}{ ORIF } & \multicolumn{2}{l|}{ MIT with the 2-point-distractor } & \multicolumn{2}{l|}{ K-Wire fixation or another combination } & Total \\
\hline Year & N & $\%$ per year & N & \% per year & N & \% per year & N \\
\hline 2007 & 1 & $5.3 \%$ & 16 & $84.7 \%$ & 2 & $10.5 \%$ & 19 \\
\hline 2008 & 3 & $13.6 \%$ & 17 & $77.3 \%$ & 2 & $9.1 \%$ & 22 \\
\hline 2009 & 0 & $0.0 \%$ & 27 & $90.0 \%$ & 3 & $10.0 \%$ & 30 \\
\hline 2010 & 2 & $8.0 \%$ & 19 & $76.0 \%$ & 4 & $16.0 \%$ & 25 \\
\hline 2011 & 0 & $0.0 \%$ & 24 & $96.0 \%$ & 1 & $4.0 \%$ & 25 \\
\hline 2012 & 0 & $0.0 \%$ & 20 & $87.0 \%$ & 3 & $13.0 \%$ & 23 \\
\hline 2013 & 1 & $4.5 \%$ & 17 & $77.3 \%$ & 4 & $18.2 \%$ & 22 \\
\hline 2014 & 0 & $0.0 \%$ & 24 & $88.9 \%$ & 3 & $11.1 \%$ & 27 \\
\hline 2015 & 0 & $0.0 \%$ & 18 & $94.7 \%$ & 1 & $5.3 \%$ & 19 \\
\hline Total & 7 & $3.3 \%$ & 182 & $85.8 \%$ & 23 & $10.8 \%$ & 212 \\
\hline
\end{tabular}

MIT: Minimally invasive technique; ORIF: Open reduction and internal Fixation; KW: Kirschner-Wire

Table 2: Annual distribution of the operative calcaneus fractures methods at the Traumacenter from 2007 - 2015. Almost the entire patient collective was treated by the minimally invasive technique with the 2 -point distractor ( $85.8 \%$ versus $10.8 \%)$, which represents a large study collective and indicates the effectiveness of this technique. 


\begin{tabular}{|llllll|}
\hline & \multicolumn{2}{c}{ Deep infection - MIT } & \multicolumn{2}{c|}{ Deep infection - ORIF + KW } & Total \\
\hline Year & $\mathrm{N}$ & $\%$ per year & $\mathrm{N}$ & $\%$ per year & $\mathrm{N}$ \\
\hline 2007 & 1 & $5.3 \%$ & 0 & $0.0 \%$ & 1 \\
\hline 2008 & 0 & $0.0 \%$ & 1 & $4.5 \%$ & 1 \\
\hline 2009 & 0 & $0.0 \%$ & 1 & $3.3 \%$ & 1 \\
\hline 2010 & 1 & $4.0 \%$ & 1 & $4.0 \%$ & 2 \\
\hline 2011 & 1 & $4.0 \%$ & 1 & $4.0 \%$ & 2 \\
\hline 2012 & 1 & $4.3 \%$ & 0 & $0.0 \%$ & 1 \\
\hline 2013 & 0 & $0.0 \%$ & 1 & $4.5 \%$ & 1 \\
\hline 2014 & 0 & $0.0 \%$ & 0 & $0.0 \%$ & 0 \\
\hline 2015 & 1 & $5.3 \%$ & 0 & $0.0 \%$ & 1 \\
\hline Total & 5 & $2.7 \%$ & 5 & $16.7 \%$ & \\
\hline
\end{tabular}

MIT: Minimally invasive technique; ORIF: Open reduction and internal fixation; KW: Kirschner-Wire

Table 3: Overview of the occurrence of complications regarding deep infections in the operative patients care. A total collective rate of $2.7 \%$ versus $16.7 \%$ could be observed, representing a low complication rate in the minimally invasive treatment. The modified technique showed that severe wound complications are less likely to occur after the minimally invasive procedure compared to ORIF or/and additional KW.

\begin{tabular}{|lllllll|}
\hline \multicolumn{2}{r}{ Necessary implant removal } & \multicolumn{2}{c|}{ Elective implant removal } & \multicolumn{2}{c|}{ Secondary Subtalar Arthrodesis } \\
Year & $\mathrm{N}$ & \% per year & $\mathrm{N}$ & $\%$ per year & $\mathrm{N}$ & $\%$ per year \\
\hline 2007 & 1 & $5.3 \%$ & 12 & $63.2 \%$ & 1 & $5.3 \%$ \\
\hline 2008 & 3 & $13.6 \%$ & 5 & $22.7 \%$ & 2 & $9.1 \%$ \\
\hline 2009 & 1 & $3.3 \%$ & 9 & $30.0 \%$ & 1 & $3.3 \%$ \\
\hline 2010 & 2 & $8.0 \%$ & 14 & $56.0 \%$ & 1 & $4.0 \%$ \\
\hline 2011 & 1 & $4.0 \%$ & 9 & $36.0 \%$ & 0 & $0.0 \%$ \\
\hline 2012 & 1 & $4.3 \%$ & 7 & $30.4 \%$ & 2 & $8.7 \%$ \\
\hline 2013 & 0 & $0.0 \%$ & 8 & $36.4 \%$ & 0 & $0.0 \%$ \\
\hline 2014 & 0 & $0.0 \%$ & 9 & $33.3 \%$ & 3 & $11.1 \%$ \\
\hline 2015 & 0 & $0.0 \%$ & 1 & $5.3 \%$ & 0 & $0.0 \%$ \\
\hline Total & 9 & $4.2 \%$ & 74 & $34.9 \%$ & 10 & $4.7 \%$ \\
\hline
\end{tabular}

Table 4: Outline of implant removals performed, splitting regarding necessary and elective implant removal. A low obligatory following operation indication of $4.2 \%$ could be observed.

Next, the rate of secondary subtalar fusions occurred with an incidence of $4.7 \%$, which represents an arthrodesis rate comparable to the existing corpus of literature data.

\section{Figures}




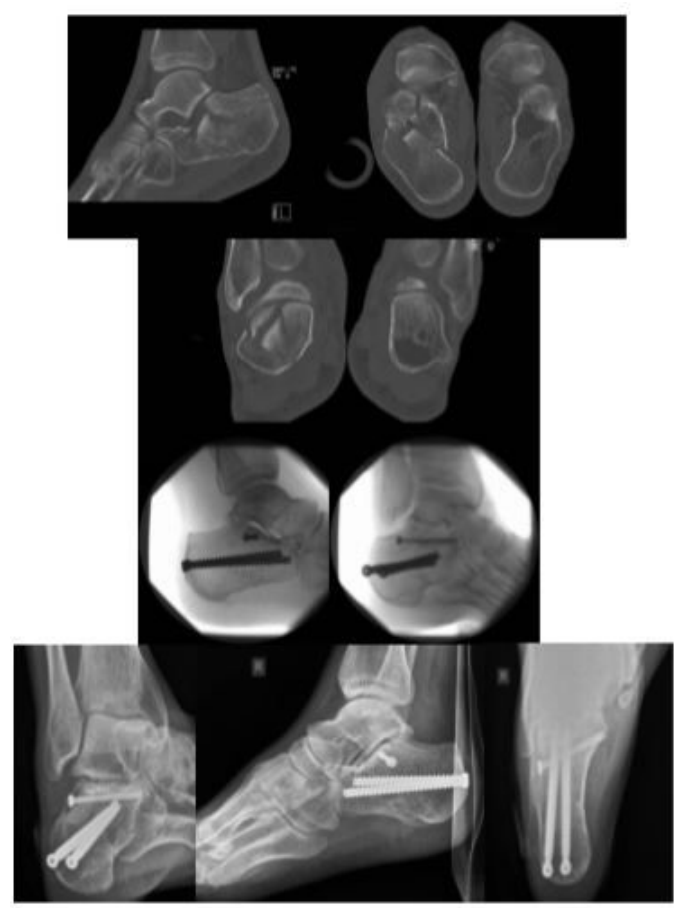

\section{Figure 1}

Patient case: A highly comminuted calcaneus fracture in depression-type form, treated by minimally invasive surgery. A case of a 42 years at operationtime, male patient, treated at the Traumacenter Linz in 2010.

\section{Supplementary Files}

This is a list of supplementary files associated with this preprint. Click to download.

- Figure8Supplement.tiff

- Figure7Supplement.tiff

- Figure6Supplement.tiff

- Figure5Supplement.tiff

- Figure4Supplement.tiff

- Figure3Supplement.tiff

- Figure2Supplement.tiff

- Figure1Supplement.tiff

- Additionalfile1SupplementTechnique.docx 